\title{
Современное развитие направления - цитологическая реактивность больного онкологического профиля
}

Институт экспериментальной патологии, онкологии и радиобиологии им. Р.Е. Кавецкого

Национальной академии наук Украины, Киев

Получено 29.11.2019

Принято в печать 04.02.2020

DOI: $10.32471 /$ clinicaloncology.2663-466X.36-4.26730

\begin{abstract}
В обзоре проанализированы данные литературы о существующих в настоящее время подходах к оценке реакции организма на опухолевый процесс на уровне соматических немалигнизированных клеток. Несмотря на различный дизайн, целью всех исследований является поиск простых неинвазивных и в то же время информативных маркеров, которые можно было бы использовать в качестве предикторов рака, для дифференциальной диагностики новообразований, прогнозирования опухолевого процесса, разработки мер профилактики, оценки эффективности лечения, а также определения чувствительности опухоли к химио- и лучевой терапии. Четко прослеживаются три основные направления исследований цитологической реактивности больного онкологического профиля. Золотым стандартом остается классический подход, позволяющий в буккальном эпителии или лимфоцитах периферической крови оценить цитогенетические, цитотоксические эффекты на основании данных, полученных при исследовании аберраций хромосом, микроядер, с недавнего времени - ДНК комет. В связи с имеющимися доказательствами того, что ключевым фактором, определяющим исход злокачественного процесса, является воспаление, широко развивается второе направление цитологической реактивности больного онкологического профиля - поиски маркеров системного воспалительного ответа на основании индексов, рассчитанных по соотношению форменных элементов крови. С недавнего времени получило развитие третье направление этой проблемы - эпигенетическое, которое заключается в изучении маркеров метилирования ДНК соматических немалигнизированных клеток больных со злокачественными новообразованиями различного генеза, в частности лимфоцитов периферической крови, а также микроРНК, ассоциированных с опухолевым ростом, для дальнейшего внедрения в клиническую практику. Имеющиеся результаты по всем трем направлениям исследований дают надежду на успешное решение в ближайшей перспективе актуальных проблем онкологии.
\end{abstract}

Ключевые слова: цитологическая реактивность больного онкологического профиля; цитогенетические, цитотоксические эффекты; буккальный эпителий; лимфоциты периферической крови; микроядра; системный воспалительный ответ; метилирование ДНК; микроРНК

\section{ВВЕДЕНИЕ}

На сегодня для большинства злокачественных новообразований остается до конца не решенной проблема ранней диагностики и прогноза течения опухолевого процесса. Исходя из этого, поиски новых подходов и других взглядов на уже существующие с позиций современных научных разработок по определению информативных показателей начальных признаков злокачественной трансформации, а также объективных критериев, по которым можно будет предсказать возникновение патологии, до сих пор являются актуальными для специалистов, работающих в области как экспериментальной, так и клинической онкологии. Прошло много времени с тех пор, как было сформулировано новое положение в онкологии «цитологическая реактивность больного онкологического профиля» и опубликована монография, в которой обобщены исследования в этом направлении, позволившие углубить фундаментальные знания об изменениях немалигнизированных, отдаленных от опухоли клеток в организме больного онкологического профиля и разработать новые биологические инструменты для применения в клинической практике [1].

Ответные реакции организма на опухоль, как и ее развитие, это многообразный, многоуровневый процесс, который отражает изменения, происходящие при злокачественной трансформации как на клеточном, так и субклеточном уровнях, а также в органах и системах, вовлеченных в канцерогенез. В частности, нарушение гомеостаза на генном уровне влечет за собой изменение функционирования различных каскадов сигнальных путей и молекул, что приводит к нарушениям иммунных процессов, метаболическим, энергетическим сдвигам и находит свое отражение в виде изменений в соматических немалигнизированных клетках, появлении опухоль-ассоциированных сывороточных маркеров, таких как С-реактивный белок, лактатдегидрогеназа, лактат, и циркулирующих в крови генетических (ДНК) и эпигенетических (микроРНК) маркеров, чему посвящены многочисленные публикации [2-7].

На сегодня цитологическая реактивность больного раком - это новый виток исследований, обогащенных разнонаправленными сведениями, полученными благодаря использованию молекулярно-биологических и молекулярногенетических биотехнологий, которые, учитывая гетерогенность новообразований, позволяют персонифицировано предсказывать прогноз заболевания и в ходе мониторинга менять стратегию лечения, а также определять эффективность проведенной терапии.

Анализ недавних данных литературы показал, что поиск простого, доступного, неинвазивного и в то же время информативного объекта исследования для клинической онкологии остановился на том же биологическом материале, соматических немалигнизированных клетках, которые применялись в прошлом веке, в частности, на лимфоцитах периферической крови и буккальном эпителии. Эти клетки признаны как субстраты, которые экспериментально проверены, объективно отражают уровень нестабильности генома, могут быть исследованы на всех этапах опухолевого процесса, включая доклинические проявления заболевания, являются удобными для проведения научных разработок, диагностики, неинвазивного скрининга, прогноза, мониторинга, определения иммунного статуса пациентов и эффективности лечения [8]. 
На сегодня в исследованиях цитологической реактивности больного онкологического профиля прослеживаются три основных направления его развития: 1 - классическое цитогенетическое; 2 - использование показателей системного воспалительного ответа; 3 - эпигенетическое с применением данных, основанных на метилировании ДНК.

\section{ЦИТОГЕНЕТИЧЕСКИЕ, ЦИТОТОКСИЧЕСКИЕ ЭФФЕКТЫ И ИЗМЕНЕНИЯ МОЛЕКУЛЯРНОГО ПРОФИЛЯ В БУККАЛЬНОМ ЭПИТЕЛИИ И ЛИМФОЦИТАХ ПЕРИФЕРИЧЕСКОЙ КРОВИ БОЛЬНЫХ РАКОМ РАЗНЫХ ЛОКАЛИЗАЦИЙ}

До настоящего времени оценка повреждающего действия на геном различных по происхождению факторов осуществляется с помощью цитогенетического метода с использованием ряда биомаркеров, которые могут быть разделены на группы: анализ хромосомных аберраций, метод ДНК-комет, микроядерный тест, измерение ДНК-аддукта $[9,10]$.

Учитывая то, что заболеваемость раком грудной железы (РГЖ) продолжает повышаться и эта патология является мультифакторной по своей природе, исследования генетической нестабильности, вызванной повреждением ДНК и неэффективностью ее репарации под воздействием различных факторов риска, являются важными как для диагностики и прогноза, так и терапевтической тактики. В связи с этим представляет интерес комплексная разработка, направленная на выяснение корреляции между социокультурными, профессиональными, эндогенными и экзогенными воздействиями на организм с уровнями генетической нестабильности с использованием микроядерного теста и анализа ДНК-комет в буккальном эпителии и лимфоцитах периферической крови женщин, больных РГЖ. Наряду с этим учитывалось проведенное лечение - химиотерапия и радиотерапия. Показано, что у пациентов старшей возрастной группы (61-80 лет) отмечался более высокий уровень показателей апоптоза (кариолиз, кариорексис), а также повреждения ДНК, по сравнению с контрольной группой лиц, с более высокими значениями у больных, которые получали химиотерапию и радиотерапию. Установлено повышение частоты цитогенетических изменений у пациенток, подвергшихся ионизирующему излучению, курящих и употребляющих алкоголь, а также корреляции между этими показателями. Последнее свидетельствует о том, что факторы риска могут вносить свой вклад в генетическую нестабильность соматических немалигнизированных клеток, возникшую во время лечения [11].

В другом исследовании, направленном на установление частоты микроядер (МЯ) у больных РГЖ, получавших химиотерапию, показано, что высокие значения этого цитогенетического теста связаны с повреждающим действием химиотерапии. Это приводит к поломкам хромосом, уменьшению числа делящихся клеток и их гибели [12].

Другой ракурс сравнительных исследований представлен на клиническом материале больных РГЖ, их родственников 1-й степени родства и здоровых лиц (120 пациентов). Оценка частоты МЯ в лимфоцитах периферической крови показала, что количество их было значительно выше по сравнению с контролем не только у больных РГЖ, но и их родственников 1-й степени. При сопоставлении данных цитогенетического исследования с клинико-патологическими характеристиками больных РГЖ установлено, что высокая частота МЯ была зарегистрирована при прогрессировании заболевания и наличии отдаленных метастазов. В целом полученные данные показали, что частота МЯ в значительной степени определяется генетическими факторами, и таким образом микроядерный тест может быть использован в качестве предиктора риска рака у лиц с отягощенным анамнезом, а, учитывая связь между частотой МЯ и метастазированием, как маркер прогноза заболевания [13]. О наследственно обусловленной нестабильности генома у больных со злокачественными ново- образованиями иного генеза свидетельствуют данные других исследователей [14].

Постепенное расширение профиля используемых методических подходов позволяет раскрыть новые возможности применения результатов исследований соматических немалигнизированных клеток в практических целях. Благодаря использованию современных молекулярных технологий было показано, что буккальный эпителий секретирует ряд сигнальных молекул, которые являются маркерами апоптоза (Р53), пролиферации (PCNA), транскрипции и дифференцировки клеток (CXCL12, WINT, RON), поэтому иммуногистохимическое исследование экспрессии указанных показателей дает объективные представления о нарушениях у больных онкологического профиля гомеостаза различных систем. Так, при исследовании уровня экспрессии CXCL12 в буккальном эпителии больных РГЖ установлено снижение этого показателя на $36 \%$ по сравнению с таковым у женщин контрольной группы, что является неблагоприятным фактом, поскольку такое изменение экспрессии молекулы CXCL12 считается пусковым механизмом онкогенеза, который стимулирует миграцию клеток и способствует метастазированию [15].

При проведении иммуногистохимического исследования экспрессии сигнальных молекул RON, CD90, CD73, CD64, Pax6, Chx10, RTF1, Oct2 и Prox1 в буккальном эпителии 50 больных РГЖ и такого же количества здоровых женщин установлено, что показатели экспрессии достоверно различались только для 8 из 9 изученных маркеров. Среди молекул кластеров дифференцировки CD90, CD73, CD64 экспрессия в буккальном эпителии у больных РГЖ снижалась для маркера CD90 - на 46\%, CD73 - на 30\% и CD64 - на 92\% по сравнению с показателями у здоровых женщин. По мнению авторов, наиболее информативными сигнальными молекулами, экспрессия которых снижается в буккальном эпителии при РГЖ, являются факторы противоопухолевой защиты организма CD64 и RON, что дает основание рекомендовать их для прогноза и коррекции лечения. Наряду с этим рекомендуется проводить исследования в буккальном эпителии маркеров терминальной дифференцировки клеток Oct2 и Chx10 [16].

Согласно данным литературы, в последнее десятилетие заболеваемость сахарным диабетом и патологические состояния в щитовидной железе возросли в геометрической прогрессии, и отмечено, что эти заболевания часто сопряжены с развитием РГЖ. Хотя между этими видами патологии нет прямой причинно-следственной связи, некоторые данные являются основанием допускать возможность каждого из этих состояний повышать риск развития РГЖ у женщин. В случае сахарного диабета инсулин, а при проблемах со щитовидной железой - тироксин могут действовать как индукторы пролиферации клеток, ведущей к развитию рака. Подтверждением этого явились результаты исследований, показавших повышенный риск РГЖ у женщин с сахарным диабетом, а также с гипертиреоидной болезнью. При цитогенетическом анализе лимфоцитов периферической крови у всех лиц в изученных группах больных был выявлен более высокий процент хромосомных аберраций по сравнению со здоровыми женщинами группы контроля [17].

О возможности применения в клинической практике фундаментальных знаний, полученных при использовании в качестве объекта исследования соматических немалигнизированных клеток, свидетельствует сравнительное исследование активности никотинамидадениндинуклеотид (НАД)- и никотинамидадениндинуклеотидфосфат (НАДФ)-зависимых дегидрогеназ лимфоцитов периферической крови у больных почечно-клеточным раком (ПКР) до проведенной операции и в динамике после хирургического лечения. Установлено, что метаболизм лимфоцитов у больных ПКР по сравнению со здоровыми лицами в дооперационный период характеризуется снижением активности пентозофосфатного цикла и глутатионзависимой антиоксидантной системы, что чревато 
снижением в них уровня реакций макромолекулярного синтеза и активации перекисных реакций. Отмечено снижение активности лактатдегидрогеназы, что свидетельствует об ингибировании анаэробного гликолиза. Наряду с этим было выявлено повышение уровня активности НАД-зависимых дегидрогеназ, которое сохранялось и в послеоперационный период. Кроме того, в этот период наблюдалось усиление синтеза аденозинтрифосфата (АТФ). Выявленные изменения пентозофосфатного цикла в лимфоцитах больных ПКР обусловливали снижение глутатионзависимой антиоксидантной защиты, которая сохранялась даже через 30 дней после лечения. Отмеченные нарушения стали основанием для заключения о том, что в послеоперационный период пациенты с ПКР нуждаются в коррекции метаболических процессов в клетках иммунной системы, и это необходимо учитывать при разработке реабилитационных программ [18].

В лимфоцитах периферической крови больных раком предстательной железы (РПЖ), который является наиболее распространенной патологией у мужчин, а также у здоровых лиц были изучены показатели повреждения генома с использованием микроядерного теста, определения аберраций хромосом, концентрации активных форм кислорода, экспрессии маркеров активации CD69, пролиферации Ki-67 и проапоптотического маркера CD95 под влиянием лучевой терапии. Также исследовано влияние гормонотерапии на свойства лимфоцитов и связь изученных показателей с эффективностью лечения, которую оценивали по уровню содержания в крови простатспецифического антигена (Prostate-Specific Antigen PSA). Показано, что радиочувствительность лимфоцитов при РПЖ коррелирует с экспрессией проапоптотического маркера CD95: при более высокой радиочувствительности экспрессия в этих клетках CD95 повышается, что свидетельствует о возможной их гибели путем апоптоза. Введение гормонов, курс которых проводили перед лучевой терапией, приводит к повышению радиочувствительности лимфоцитов при РПЖ, снижению содержания активных форм кислорода, не существенно повышенного до лечения. С точки зрения прогноза лечения больных РПЖ, по мнению авторов, важным фактом является выявленная корреляционная связь между исходной (до начала лечения) частотой клеток с МЯ и результативностью лечения: чем меньше поврежденных клеток, тем выше эффективность терапии [19].

Группой авторов в результате исследования цитогенетического статуса больных раком желудка в буккальных эпителиоцитах выявлено статистически достоверное увеличение доли клеток с МЯ в 8 раз, с протрузиями - в 4,3 раза, при этом суммарное количество клеток с этими аномалиями в 6,2 раза превышало таковое у здоровых лиц. Кроме того, установлено увеличение количества клеток с показателями, косвенно отражающими пролиферацию: рост в 2,7 раза доли клеток с двумя ядрами и в 2,4 раза общей суммы клеток с двумя и большим количеством ядер у больных раком по сравнению с группой контроля. После радикального лечения уровни указанных кариологических показателей снижались, что, по мнению авторов, свидетельствует об их прогностической значимости [20].

У 155 больных с лейкоплакией слизистой оболочки полости рта с разной степенью гиперкератоза, согласно классификации ВОО3 (2005 г.), была проведена оценка эффективности использования микроядерного теста в клетках буккального эпителия для ранней диагностики этой патологии. Показано, что МЯ, появление которых вызвано нарушением дифференцировки эпителиоцитов, выявлены у пациентов с разной степенью выраженности гиперкератоза (как при CIN2, так и CIN3, с разницей лишь в 1,3 раза $(\mathrm{p}<0,05))$. Это свидетельствует о том, что в обоих случаях существует вероятность возникновения злокачественной трансформации буккальных эпителиоцитов. Таким образом, показано, что на фоне общего повышения пролиферативной активности эпителиоцитов буккального эпителия по мере распространения плоскоклеточной внутриэпителиальной неоплазии (Squamous Intraepithelial Neoplasia - SIN) для каждой исследуемой группы характерно появление в клетках МЯ. Причем частота встречаемости МЯ повышалась по мере роста гиперплазии, что свидетельствует о повышении вероятности малигнизации и развития рака слизистой оболочки полости рта у пациентов с лейкоплакией SIN3 [21]. Эти данные подтверждаются результатами исследований патологических состояний в слизистой оболочке полости рта, проведенных другими авторами [22].

Поскольку рак мочевого пузыря (РМП) является одним из наиболее агрессивных злокачественных новообразований мочевыводящих путей и самым высоко затратным в плане лечения, необходимы неинвазивные, чувствительные молекулярные биомаркеры для оптимизации диагностики и мониторинга этой патологии. В этом аспекте проведено исследование, направленное на оценку репаративной способности ДНК после повреждения в лимфоцитах периферической крови 146 пациентов с РМП и 155 лиц контрольной группы с использованием модифицированного анализа ДНК-комет, дающего возможность оценить активность репарации ДНК после воздействия на клетки бенз(а)пирен диолэпоксида. В результате исследования была выявлена связь между уровнями повреждения ДНК в лимфоцитах периферической крови при РМП и исходом заболевания. Повреждение ДНК в лимфоцитах было значительно выше при менее выраженной степени дифференцировки опухолей больных. Установлено, что общая выживаемость пациентов также зависела от степени повреждения ДНК в лимфоцитах. Продолжительность жизни пациентов была ниже у лиц с высокими показателями повреждения ДНК (отношение рисков (ОР) 3,7; $95 \%$ доверительный интервал (ДИ) 1,3-10,6; p=0,02), поэтому, по мнению исследователей, этот тест может быть маркером выживаемости больных РМП [23].

Значительный интерес представляют данные, полученные на клиническом материале 106 больных раком эндометрия (РЭ), у которых было проведено исследование нестабильности генома в лимфоцитах периферической крови и параллельно в опухолевых клетках методом ДНК-комет с учетом клинических и морфологических характеристик. В качестве контроля использованы результаты исследования с участием 30 здоровых женщин. Установлено, что степень повреждения ДНК в лимфоцитах больных РЭ была в 2,2 раза выше, чем у здоровых лиц $(8,3 \pm 0,7$ и $3,7 \pm 0,4 \%$ ДНК в хвосте комет соответственно) $(\mathrm{p}<0,05)$. При этом не было выявлено связи между уровнями повреждения ДНК в опухолевых клетках эндометрия и в лимфоцитах периферической крови $(\mathrm{r}=0,11 ; \mathrm{p}>0,05)$. Величины повреждения ДНК как в опухолевых клетках, так и в лимфоцитах периферической крови не были связаны со степенью дифференцировки опухоли, а также с глубиной инвазии в миометрий, но зависели от индекса массы тела (ИМТ) пациенток с РЭ: высокий уровень повреждений наблюдался у пациенток с повышенными значениями ИМТ. Показатели повреждения ДНК в опухолевых клетках больных РЭ были выше у лиц с семейной агрегацией рака по сравнению с лицами со спорадически возникшими опухолями ( $32,3 \pm 2,9$ и $22,8 \pm 1,8 \%$ ДНК в хвосте комет соответственно) $(\mathrm{p}<0,05)$. У женщин с высоким уровнем повреждения ДНК в лимфоцитах периферической крови риск РЭбыл выше, чем у лиц с низким уровнем повреждения. Таким образом, высокий уровень повреждения ДНК лимфоцитов периферической крови больных РЭ как показатель нестабильности генома, повышенные значения ИМТ, семейная история рака в совокупности могут быть расценены как факторы риска развития онкопатологии и их следует учитывать при обследовании женщин с гиперпластическими процессами в эндометрии [24].

В последние годы для прогнозирования риска развития рака у лиц, связанных с влиянием на организм длительно существующих вредных привычек (курение, алкогольная зависимость), с действием факторов внешней среды, про- 
фессионального вреда широко используется цитогенетическая характеристика ядер соматических немалигнизированных клеток - образование МЯ вследствие повреждения ДНК, гибель клеток, пикноз, кариолиз и кариорексис [25-32].

Таким образом, анализ данных литературы свидетельствует о целесообразности использования в онкологии в качестве диагностического материала соматических немалигнизированных клеток для выявления в них цитогенетических и цитотоксических эффектов, метаболических и молекулярно-биологических изменений, являющихся отражением нарушений в органах и системах организма на различных этапах онкогенеза, с целью формирования групп риска, прогнозирования опухолевого процесса и контроля эффективности лечения.

\section{ВОСПАЛЕНИЕ И РАК: ИЗМЕНЕНИЯ СООТНОШЕНИЯ ФОРМЕННЫХ ЭЛЕМЕНТОВ ПЕРИФЕРИЧЕСКОЙ КРОВИ БОЛЬНЫХ КАК СИСТЕМНАЯ ИММУННАЯ РЕАКЦИЯ ОРГАНИЗМА}

В связи с трудностями, возникающими при диагностике и прогнозировании клинического течения большинства злокачественных новообразований, прослеживается тенденция к отходу многих исследований от классических цитогенетических методов (оценка гено- и цитотоксических эффектов, анализ хромосомных аберраций, подсчет количества ДНК в хвосте комет), поскольку появилось все больше доказательств того, что связанное с раком воспаление является ключевым фактором, определяющим исход заболевания у пациентов с раком. В течение последнего десятилетия были исследованы различные маркеры воспаления в попытке использовать их в клинической практике для диагностики, лечения больных онкологического профиля и прогноза заболевания. С появлением убедительных доказательств роли воспаления в биологии рака постулируется наличие системного воспалительного ответа как имеющего прогностическое значение.

Одним из обычно доступных маркеров системного воспалительного ответа является показатель соотношения нейтрофилов и лимфоцитов (Neutrophil to lymphocyte ratio - NLR), полученный в результате подсчета абсолютного количества нейтрофилов и лимфоцитов по данным общего анализа крови $[33,34]$.

Согласно данным литературы, связанное с раком воспаление возникает в микроокружении опухоли, а также в системном кровообращении и коррелирует с прогрессированием заболевания и прогнозом при многих видах рака. Иммунные медиаторы принимают участие на разных этапах развития и прогрессирования рака, включая канцерогенез, рост опухоли, инвазию опухоли и метастазирование, и, в свою очередь, злокачественно трансформированные клетки могут рекрутировать и активировать иммунные клетки посредством хемокинов и простагландинов. Количество циркулирующих клеток крови, в том числе нейтрофилов, лимфоцитов, тромбоцитов, и уровни циркулирующих белков, включая C-реактивный белок и интерлейкины, связанные с воспалительными реакциями, являются ключевыми факторами в онкогенезе и могут предоставить ценную информацию для улучшения стратификации риска заболевания раком и более целенаправленной помощи пациентам [35].

В настоящее время предложен целый ряд коэффициентов, индексов, основанных на количественных соотношениях форменных элементов крови, представленных в общем анализе крови.

Так, проведено популяционное когортное исследование лиц в возрасте старше 45 лет (8024 человека), направленное на установление связи между так называемым системным индексом иммунного воспаления (Systemic immune-inflammation index - SII) и случаями возникновения рака. Связь между SII и риском солидного рака любой локализации во время наблюдения была оценена с использованием модели пропорциональных рисков Кокса. В течение максимального периода наблюдения - 10,7 лет у 733 человек на основании SII был диагностирован рак. Высокие значения SII в начале исследования, связанные с риском развития солидных форм рака, после поправки на возраст, пол, социально-экономический статус, курение, ИМТ и сахарный диабет II типа повысились на $30 \%$ (суммарный коэффициент опасности 1,30 [95\% ДИ 1,11-1,53]). Риск развития рака был постоянным в течение мониторинга, но более высоким у лиц с длительным действием факторов. Результаты исследования продемонстрировали, что высокий SII является информативным и независимым индикатором риска развития солидного рака [36].

У лиц с патологией придатков (306 наблюдений), в том числе больных раком яичников (РЯ), проведена оценка связи между NLR, а также соотношением тромбоцитов и лимфоцитов (Platelet/Lymphocyte Ratio - PLR) в предоперационный период. Установлено, что у пациентов с РЯ были самые высокие значения NLR и PLR ( $<<0,05$ и $\mathrm{p}<0,001$ соответственно). Многофакторный анализ показал, что более высокие значения NLR и PLR, позволившие предсказать РЯ при патологии придатков, соответствовали 3,35 для NLR при чувствительности $55 \%$ и специфичности $81 \%$ (95\% ДИ 0,544-0,752; p<0,05), при предельном значении - 572,9 для PLR при чувствительности $100 \%$ и специфичности $0,38 \%$ (95\% ДИ 0,192-0,381; $\mathrm{p}=0,001)$. Полученные результаты стали основанием полагать, что значения NLR и PLR могут способствовать выявлению PЯ у пациентов с патологией придатков в дооперационный период [37].

Другими учеными в группе пациентов, состоящей из 190 больных РЯ, было проведено исследование, направленное на выявление предикторов общей и безрецидивной выживаемости с использованием большого арсенала маркеров, включающих уровни фибриногена, альбумина, С-реактивного белка (СРБ) и сывороточного антигена-125 (СА-125), а также NLR и соотношение тромбоцитов/лимфоцитов (Platelet/Lymphocyte Ratio - PLR). У пациентов c PLR $>203$ отмечали более короткие сроки жизни без прогрессирования процесса и общей выживаемости, чем у лиц с PLR $\leqslant 203$ (11 против 24 мес и 28 против 64 мес). Однофакторный анализ показал, что степень дифференцировки, послеоперационная масса остаточной опухоли, асцит и уровни всех маркеров системного воспалительного ответа коррелировали с выживаемостью без прогрессирования процесса и с общей выживаемостью. Многофакторный анализ позволил убедиться, что PLR был независимо связан с выживаемостью без прогрессирования (ОР 1,852; 95\% ДИ $1,271-2,697 ; \mathrm{p}=0,001)$ и общей выживаемостью (ОР 2, 158; $95 \%$ ДИ 1,468-3,171; $<0,001)$, а также степенью дифференцировки и послеоперационной массой остаточной опухоли. У пациентов с PLR $>203$ была отмечена более выраженная склонность к прогрессированию опухолевого процесса $(\mathrm{p}=0,002)$, увеличению послеоперационной остаточной массы опухоли $>2 \mathrm{~cm}(\mathrm{p}=0,032)$, асциту $(\mathrm{p}<0,001)$ и повышению числовых значений других маркеров системного воспалительного ответа $(\mathrm{p}<0,001)$. В целом исследование показало, что PLR превосходит другие маркеры воспаления (CA-125, NLR, фибриноген, СРБ и альбумин) и может служить в качестве предиктора выживаемости у пациентов с РЯ [38].

Группой авторов изучена эффективность использования NLR для диагностики опухоли иного генеза - почечно-клеточного рака (ПКР) - в проспективном и ретроспективном исследовании. Изучены 2 группы пациентов с доброкачественными опухолями почек и хроническим пиелонефритом, а также больные с ПКР. NLR составляло 1,97 для 1-й группы и 2,35 - для 2-й, и разница между этими показателями была статистически достоверной ( $<<0,0001)$. По мнению авторов, 
эти данные свидетельствуют о том, что NLR может быть одним из диагностических параметров ПКР в предоперационный период [39].

Изучение другого маркера системного воспалительного ответа периферической крови - соотношения лимфоцитов и моноцитов (lymphocyte to monocyte ratio - LMR), рассчитанного на основании абсолютных величин этих форменных элементов крови, подтвердили значение данного показателя как независимого прогностического фактора у 474 пациентов с аденокарциномой поджелудочной железы. Высокие значения LMR aссоциировались с общеизвестными прогностическими факторами, включая стадию опухолевого процесса и степень дифференцировки опухоли ( $<<0,05)$, и при однофакторном анализе оказались значимым фактором лучшей выживаемости пациентов с этой патологией (суммарный коэффициент опасности 0,$70 ; 95 \%$ ДИ 0,57-0,85; p $<0,001)$. Благодаря многофакторному анализу, включающему возраст, индекс Карнофски, степень дифференцировки опухоли, стадию процесса, проведенную химиотерапию, LMR и хирургическую резекцию, было доказано, что высокие значения LMR являются независимым прогностическим фактором по отношению к выживаемости больных (суммарный коэффициент опасности $-0,81 ; 95 \%$ ДИ 0,66-0,99; $\mathrm{p}=0,04)$ [40]

Оценка прогностической значимости 2 маркеров системного воспалительного ответа: NLR, PLR, а также абсолютного числа нейтрофилов и лимфоцитов, у 251 больного раком носоглотки подтвердила результаты исследований, проведенных другими авторами у больных с иной локализацией рака, свидетельствующие о том, что NLR и PLR в предоперационный период могут служить независимыми прогностическими маркерами [41].

На клиническом материале 1623 пациентов, перенесших резекцию по поводу колоректального рака (КР), изучена прогностическая значимость одного из маркеров системного воспалительного ответа - LMR. Многофакторный анализ показал, что высокие значения LMR коррелировали с лучшей общей выживаемостью больных (ОР 0,569; 95\% ДИ 0,478-0,677; $\mathrm{p}<0,001)$ независимо от возраста $(\mathrm{p}<0,001)$, Т-стадии $(\mathrm{p}<0,001)$, $\mathrm{N}$-стадии $(\mathrm{p}<0,001)$. Оказалось, что NLR, PLR, комбинированный статус BRAF-MMR и другие изученные прогностические маркеры не были независимо значимыми факторами. В многофакторном анализе LMR оставался единственным независимым предиктором (OP 0,620; 95\% ДИ 0,437-0,880; $\mathrm{p}=0,007)$ общей выживаемости больных КР, который существенно превосходит ранее существовавшие биомаркеры [42].

Для проверки прогностической значимости NLR в периферической крови мужчин, больных РПЖ, которым предстояла радикальная простатэктомия, были обследованы 389 пациентов с усредненными показателями: по возрасту 65 лет, периоду наблюдения - 51,5 мес, безрецидивной выживаемости - 51,3 мес. Исследование показало, что пациенты, у которых количество нейтрофилов в периферической крови превышало $60 \%$, а общее число лимфоцитов было меньше 1500 , отличались высокой частотой возникновения рецидивов. Не установлена корреляция между числовыми значениями NLR и исходным уровнем PSA, а также количеством баллов по Глисону и стадией опухолевого процесса. В многофакторном анализе, включающем возраст больных, уровень PSA и NLR, установлено, что NLR является наиболее важным прогностическим фактором возникновения рецидивов [43].

Другое исследование с фундаментальным аспектом проведено у 994 больных РПЖ с радикальной простатэктомией, в котором использован широкий спектр иммуногистохимических маркеров для оценки связи между маркером системного воспаления NLR (рассчитан в предоперационный период), клинико-патологическими характеристиками, молекулярным профилем первичной опухоли. В частности, в опухолях проведена иммуногистохимическая оценка экспрессии ан- дрогенного рецептора (androgen receptor - AR), ERG, PTEN, p-AKT, Bcl-2, Beclin-1, Ki-67, Cluster of Differentiation (CD) 3, $\mathrm{CD} 4, \mathrm{CD} 8$, интерферона- $\gamma$ (Interferon- $\gamma-\mathrm{IFN}-\gamma)$ и фактора некроза опухоли (Tumor necrosis factor $\alpha-\mathrm{TNF}-\alpha$ ). Анализ полученных данных показал, что существенных различий при сопоставлении числовых значений маркера NLR, pacсчитанных до проведенной операции, с клинико-патологическими характеристиками больных РПЖ не наблюдалось ( $>0,05)$. В группе больных с высоким значением NLR (NLR $\geqslant 2$ ) определялось большее число опухолей с инвазией капсулы простаты $(p=0,047)$. Однако не было выявлено значительной корреляции между NLR и инфильтрирующими опухоль $\mathrm{CD} 3^{+}$клетками, соотношением CD4/CD8, AR, ERG, PTEN, p-AKT, Bcl-2, Beclin-1, Ki-67, IFN- $\gamma$ или TNF- $\alpha(\mathrm{p}>0,05)$. Оценка данных без учета послеоперационной адъювантной гормональной и лучевой терапии с использованием одно- и многофакторного анализа показала, что в группе больных с высоким NLR общая выживаемость была значительно ниже ( $<<0,05)$, чем с низким. На основании отмеченных особенностей авторы считают, что хотя NLR не отражает всех характеристик РПЖ и состояния микроокружения опухоли, высокий NLR в периферической крови может служить независимым предиктором лучшей выживаемости пациентов [44].

Наряду с оценкой значимости для урологической практики NLR периферической крови исследуются другие маркеры системного воспаления. Проведено сопоставление PLR в периферической крови у 155 здоровых лиц, 168 пациентов с доброкачественной гиперплазией предстательной железы и 103 больных РПЖ для установления прогностической ценности маркера PLR у пациентов с РПЖ. Все больные были разделены на группу с низким значением PLR (PLR $<150)$ и группу с высоким значением PLR (PLR $\geqslant 150)$ для сравнительного изучения этих показателей в зависимости от клинико-патологических характеристик. Установлены существенные статистически значимые различия между пациентами групп сравнения. Кроме того, в группе с высоким значением PLR отмечено увеличение количества тромбоцитов, нейтрофилов, уровня PSA, лактатдегидрогеназы, СРБ и щелочной фосфатазы при снижении уровня гемоглобина. 3-летняя выживаемость в группе лиц с высокими показателями PLR была значительно ниже. В целом анализ 3-летней выживаемости в группах больных с высокими и низкими значениями PLR по отношению к другим изученным характеристикам явился основанием расценивать этот показатель как независимый прогностический критерий для исследуемой категории больных [45].

Помимо оценки ряда показателей системного воспалительного ответа (лимфоциты, нейтрофилы, моноциты, LMR, отношение нейтрофилов к моноцитам (neutrophil to monocyte ratio - NMR) и NLR) как предикторов у больных раком различных локализаций рядом исследователей проводился анализ прогностической значимости их после проведенного лечения. В частности, было предпринято ретроспективное исследование когорты женщин, больных РГЖ (150 наблюдений), получавших неоадъювантную химиотерапию. Установлено, что высокие показатели $\operatorname{LMR}(\geqslant 5,46)$ и низкие $\operatorname{NLR}(<3,33)$ сопряжены с меньшим процентом рецидивов ( $\mathrm{p}=0,048$ и $\mathrm{p}=0,015$ соответственно). Также отмечено, что NLR ассоциировался с лучшей выживаемостью больных $(\mathrm{p}=0,024)$. Полученные данные свидетельствуют о том, что высокий LMR и низкий NLR можно рассматривать как благоприятные прогностические факторы у больных РГЖ, получавших неоадъювантную химиотерапию [46].

В другом исследовании, проведенном у больных с ранним и местно-распространенным РГЖ, получавших неоадъювантную химиотерапию, авторы поставили цель выяснить возможную связь между показателями системного воспаления и патоморфозом опухоли, использовав 2 показателя периферической крови - NLR и PLR. Анализ полученных 
данных показал, что при пороговых значениях для NLR и PLR 2,42 и 104,47 соответственно наблюдался наиболее выраженный эффект от проведенного лечения, когда эти показатели оценивались в сочетании. При раздельной оценке патоморфоза по этим величинам NLR и PLR достоверная связь между сравниваемыми показателями не отмечена. Однако при сочетании NLR и PLR у пациентов с низким профилем (NLRlow/PLRlow) отмечался более высокий уровень патоморфоза, чем у больных с более высокими его значениями (NLRhigh и/или PLRhigh). Прогностическая ценность NLRlow/PLRlow не зависела от общеизвестных прогностических факторов: клинических, Ki67 и молекулярных подтипов. Показано, что комбинация NLR и PLR у больных РГЖ может отражать реактивные изменения на уровне организма, а низкие значения NLR и PLR могут указывать на состояние активации иммунной системы, что в целом дает возможность предсказать степень патоморфоза у пациентов с РГЖ, получавших неоадъювантную химиотерапию [47].

Особо информативным, современным по дизайну и важным с точки зрения понимания взаимосвязи между опухолью и организмом является исследование, проведенное на клиническом материале 684 больных РГЖ, в котором авторы стремились установить наличие связи между количеством иммунных клеток в периферической крови и инфильтрирующих опухоль лимфоцитов в ее микроокружении. При анализе полученных результатов в 2 группах больных с так называемыми лимфоцит-преобладающими опухолями - $99(17,2 \%)$ и не содержащими в микроокружении лимфоцитов $478(82,8 \%)$ оказалось, что из 3 изученных маркеров системного воспаления (количество лимфоцитов, нейтрофилов, NLR), лимфоцитсодержащие опухоли характеризовались более низким количеством нейтрофилов в периферической крови по сравнению с таковым в опухолях, не содержащих в микроокружении лимфоцитов (3330 против 3660; p=0,004). Другие показатели не имели существенных отличий. Поэтому уменьшение количества нейтрофилов в периферической крови было признано независимым клиническим показателем для прогнозирования течения опухолевого процесса у больных с лимфоцит-преобладающим РГЖ (ОР 0,736; $95 \%$ ДИ 0,591-0,917; p=0,004). В результате авторы установили, что низкое количество нейтрофилов в периферической крови характерно для РГЖ с преобладанием в опухолевой ткани лимфоцитарных инфильтратов, и это явилось подтверждением гипотезы о существовании связи между количеством циркулирующих в крови иммунных клеток и выявляемыми в микроокружении опухоли [48].

Таким образом, многочисленные данные литературы, полученные на основании оценки соотношения лимфоидных элементов в периферической крови и, в частности, нейтрофилов и лимфоцитов у больных онкологического профиля, показали, что это простой, доступный подход к диагностике рака, который может стать предикторно-скрининговым для широкого применения в клинической практике. Учитывая сказанное, а также то, что первым этапом обследования больных является посещение семейного врача, который, как правило, направляет пациента на общий анализ крови, предусматривающий количественное определение в ней форменных элементов, целесообразным является акцентировать внимание врачей-лаборантов на необходимости производить расчет NLR на основании общего количества таких клеток. Это представляет особую важность, в первую очередь в отношении больных с длительно текущими хроническими процессами, в частности, желудочно-кишечного тракта и легких. Возможно, такая тактика в какой-то мере позволит упредить развитие запущенных случаев рака, так как на основании данных исследования крови при неблагоприятном результате пациент будет направлен семейным врачом для консультации к онкологу.

\section{ЭПИГЕНЕТИЧЕСКИЕ ПОДХОДЫ ДЛЯ ОЦЕНКИ ИЗМЕНЕНИЙ В СОМАТИЧЕСКИХ НЕМАЛИГНИЗИРОВАННЫХ КЛЕТКАХ БОЛЬНЫХ ОНКОЛОГИЧЕСКОГО ПРОФИЛЯ}

На начальных этапах открытий в области молекулярной биологии, связанных с исследованиями рака, основные усилия были направлены на выявление генетических изменений, произошедших в последовательности ДНК. Это способствовало расширению представлений о патогенезе и биологии рака и открыло новые горизонты для использования фундаментальных знаний о возникновении злокачественного роста в клинической онкологии. Однако не меньшее значение имеет изучение тех звеньев онкогенеза, которые не связаны с нарушением последовательности ДНК, а затрагивают эпигенетические аспекты этой проблемы. Спектр возможностей исследований в этой области охватывает изменения в метилировании цитозинов в ДНК, изменение в структуре гистонов и хроматина [49, 50].

Метилирование ДНК является определяющим звеном при проведении эпигенетических исследований, позволяющим дифференцировать различные подтипы лейкоцитов, и составляет альтернативу цитологическим исследованиям при оценке профилей лейкоцитов [51, 52]. Имеются данные о том, что дифференциально метилированные участки (differentally methylated regions - DMR) в ДНК, выделенной из цельной крови, можно использовать для оценки соотношений циркулирующих подтипов лейкоцитов. Для описания этих DMR в целях изучения профилей лейкоцитов предложен термин «иммунометиломика».

Коллективом авторов была поставлена и решена широкомасштабная задача - создать биоинформационный инструмент для оценки значимости NLR с использованием метилирования ДНК и определения его диагностических и прогностических характеристик в популяциях людей со злокачественными новообразованиями различного генеза. Разработан индекс NLR (mdNLR), полученный на основании метилирования ДНК с использованием нормальных изолированных библиотек метилирования лейкоцитов. Этот метод был применен при исследовании РМП, рака головы и шеи, яичников и РГЖ, а также по отношению к лицам, у которых не были выявлены злокачественные опухоли. У больных с наличием злокачественных новообразований индексы mdNLR были выше по сравнению с такими в группе контроля и ассоциировались с повышенным риском смерти: высокие значения mdNLR (>5) являлись показателями низкой выживаемости. Отмечена взаимосвязь между курением и выживаемостью больных раком. В целом полученные результаты позволили авторам высказаться о том, что индекс NLR, основанный на метилировании ДНК изученных лейкоцитов больных со злокачественными новообразованиями разных локализаций, по отношению к NLR лиц без онкологической патологии, может быть использован клиницистами в онкологической практике для оценки рисков возникновения рака и выживаемости больных, а также при проведении эпидемиологических исследований. Авторы также считают, что поскольку цитологическое определение NLR не всегда возможно (при наличии архивной крови), mdNLR, рассчитанный с учетом метилированной ДНК лейкоцитов, является более информативным для проведения ассоциативных исследований [52].

На основании результатов метилирования ДНК периферической крови больных с глиомой были выделены подтипы лейкоцитов и рассчитано NLR. Проведенные сопоставления показали, что величина NLR выше у больных с глиомой по сравнению с группой контроля, и выживаемость таких больных была ниже при mdNLR श4,0 в моделях пропорциональных рисков Кокса с поправкой на возраст, пол, степень дифференцировки опухоли и молекулярный подтип (суммарный коэффициент опасности 2,02; $95 \%$ ДИ 1,11-3,69). В результате метилирования ДНК лимфоцитов больных с глиомой выявлены 5 локусов 
CpG. Каждый из этих локусов ассоциировался с выживаемостью и открывал возможность для использования при исследованиях свежих или архивных образцов периферической крови. Авторы считают, что mdNLR (основанный на метилировании ДНК) является новым потенциальным биомаркером метилирования, который может быть использован в клинической практике [53].

Поскольку после радикально проведенной резекции опухоли у больных с плоскоклеточным раком полости рта клиницисты отмечают высокую частоту возникновения рецидивов и метастазов, у 40 больных с этим диагнозом было проведено сравнительное исследование, нацеленное на определение молекулярных изменений в самой опухоли, ее краях и визуально неизмененной слизистой оболочке для оценки возможности использования в качестве прогностических маркеров генной амплификации с-Мус и HER2 и метилирования промотора p14 и p16. Полученные данные свидетельствуют о том, что в опухолевой ткани наблюдаются наиболее выраженные изменения, и их отмечают чаще, чем в визуально неизмененной слизистой оболочке полости рта ( $\mathrm{p}<0,001$ и $\mathrm{p}=0,027$ соответственно). Амплификация HER2 в краях опухоли $(\mathrm{p}<0,001)$, a также наличие других изменений в этих участках $(p=0,001)$ и макроскопически неизмененной слизистой оболочке полости рта $(\mathrm{p}<0,001)$ коррелировали с выживаемостью больных плоскоклеточным раком [54].

Представляет интерес исследование, позволившее в слизистой оболочке полости рта 400 женщин, больных раком различных локализаций, выявить $1501 \mathrm{CpG}$ сайт, уровень метилирования ДНК в которых коррелировал с продолжительностью курения, и на основании этого разработать индекс курения, позволяющий отличить неизмененную ткань от пораженной раком. Эти данные были подтверждены в независимом исследовании при участии 390 женщин. В peзультате сопоставления количества метилированных сайтов в слизистой оболочке полости рта и клетках крови больных со злокачественными новообразованиями различного генеза отмечено, что их число в буккальном эпителии в 40 раз превышало таковое в клетках крови, что явилось основанием для авторов рекомендовать для проведения дифференциальнодиагностических исследований использовать буккальный эпителий [55].

В последние годы в онкологии в центре внимания научных исследований, направленных на выяснение роли эпигенетических нарушений в возникновении рака, находятся молекулы микроРНК. Это связано с тем, что микроРНК расцениваются как малые, консервативные, некодирующие последовательности РНК и как один из ключевых факторов в посттранскрипционных механизмах регуляции генов. В зависимости от выполняемой функции они могут либо стимулировать процессы пролиферации, дифференцировки и метаболизма, либо действовать в качестве супрессоров, а также влиять на программу апоптотической гибели клеток и злокачественной трансформации [56]. На сегодня результаты исследования микроРНК в опухоли, а также в циркулирующей крови используются в целях диагностики, прогноза и лечения больных со злокачественными новообразованиями [57-60]. Эра использования этих молекул для оценки цитологической реактивности больного онкологического профиля только начинается [58]

Изучен ряд miRNA и mRNA и профили их экспрессии в лимфоцитах периферической крови 74 больных семейным РЯ и 47 лиц группы контроля. В их числе были 6 супрессорных miRNAs и 1 активная miRNAs. Показано, что hsa-miR-34b играет ключевую роль в этом комплексе и взаимодействует с другими miRNA. Также установлено, что hsa-miR-136 и hsamiR-335 связаны с p53 и Erk1/2 сигнальными путями и опухолевыми супрессорами, в частности PTEN. На основании полученных данных авторы считают, что эти микроРНК могут служить новыми инструментами для изучения особенностей транскрипции в соматических немалигнизированных клетках лиц с семейным РЯ [61].

Многие исследователи отмечают, что, несмотря на большую значимость выявляемых при развитии рака нарушений регуляторных процессов в опухолевой ткани на эпигенетическом уровне, в том числе с участием микроРНК, состояние этих эпигенетических маркеров в лимфоидных элементах периферической крови до сих пор мало изучено. Поэтому сделана попытка охарактеризовать дифференциально экспрессируемые микроРНК моноцитов крови, полученных от больных раком желудка, РГЖ и здоровых доноров. Показана повышенная экспрессия 74 miRNAs и сниженная 46 miRNAs в моноцитах пациентов с РГЖ и раком желудка по сравнению со здоровыми донорами. Это позволило авторам предположить, что экспрессия 120 miRNAs в клетках крови больных с изученной патологией обусловлена наличием опухолевого процесса. 38 микроРНК были подвергнуты дополнительной проверке с использованием количественной полимеразной цепной реакции в реальном времени. Установлено, что 36 из 120 экспрессированных микроРНК у больных РГЖ и раком желудка связаны с генами, ассоциированными с онкогенезом, и вовлечены в соответствующие сигнальные пути, в частности HIF-1 и mTOR. Проведенное фундаментальное исследование на моноцитах периферической крови позволило расширить представление о роли микроРНК во взаимосвязи между опухолью и соматическими немалигнизированными клетками организма больного онкопатологией [62].

Имеются сообщения, в которых авторы с помощью анализа профиля микроРНК в слизистой оболочке полости рта, пораженной раком, попытались выяснить возможность использования этого эпигенетического маркера в прогностических целях. В одном из исследований, выполненном на архивном материале, была идентифицирована панель из 12 дифференциально экспрессированных микроРНК, 8 из которых были проверены в независимой выборке. При построении модели с использованием miR-486-3p, miR-139-5p и miR-21 оказалось, что она позволяет выявить рак в слизистой оболочке полости рта с чувствительностью $100 \%$ и специфичностью $86,7 \%$ при общей ошибке $6,7 \%$. Авторы также показали, что исследованные микроРНК как биомаркеры могут быть использованы не только при исследовании операционного материала, но и в архивных образцах [63].

Для оценки возможности использования микроРНК в качестве биомаркеров риска РГЖ у женщин был проанализирован профиль 5 микроРНК, идентифицированных как маркеры потенциального риска: miR-144-3p, miR-451a, miR-144-5p, miR-183-5p и miR-708-5p в мононуклеарных клетках периферической крови больных РГЖ и здоровых лиц. Оказалось, что экспрессия 4 из них была повышена, в то время как экспрессия miR-708-5p - снижена. Несмотря на то, что при повторном исследовании с использованием метода полимеразной цепной реакции не были получены достоверные результаты, высказано мнение о возможности использования дифференциально экспрессируемых микроРНК в мононуклеарных клетках периферической крови больных РГЖ в качестве неинвазивных биомаркеров для прогнозирования этого заболевания, а также о необходимости приведения дополнительных более масштабных исследований для подтверждения полученных данных [64].

В целом проведенный обзор данных литературы дает основание надеяться, что «реанимация» направления цитологической реактивности больного онкологического профиля, согласно современным представлениям о биологии рака, в перспективе позволит поднять на новый уровень раннюю диагностику этой патологии и реализовать концепцию персонализированного прогноза и патогенетически адекватного лечения больного онкологического профиля. 


\section{СПИСОК ИСПОЛЬЗОВАННОЙ ЛИТЕРАТУРЫ}

1. Ганина, К. П., Полищук, Л. З., Бородай, Н. В., Налескина Л. А., Исакова Л. М., Несина, И. П., ... Бучинская, Л. Г. (1995). Цитологическая реактивность онкологи ческого больного. Київ: Наукова думка.

2. Лозовская, Ю.В., Лукьянова, Н. Ю., Задворный, Т. В., Собченко, С. А., \& Чехун, В. Ф. (2016). Прогностические сывороточные биомаркеры рака молочной железы: лактофферин, лактатдегидрогеназа и с-реактивный протеин. Онкологический журнал, 10(4), 33-41. Восстановлена из https://elibrary.ru/item. asp?id=30033794

3. Link, A., \& Kupcinskas, J. (2018). MicroRNAs as non-invasive diagnostic biomarkers for gastric cancer: Current insights and future perspectives. World Journal of Gastroenterology, 24(30), 3313-3329. doi: 10.3748/wjg.v24.i30.3313

4. Mengying Cui, Hongdan Wang, Xiaoxiao Yao, Dan Zhang, Yingjun Xie, Ranji Cui, \& Xuewen Zhang (2019). Circulating MicroRNAs in Cancer: Potential and Challenge. Frontiers in Genetics, 10, 626. doi: 10.3389/fgene.2019.00626

5. Sumbal, S., Javed, A. Afroze, B. Zulfiqar, H. F., Javed, F., Noreen, S.,... ljaz, B. (2018). Circulating tumor DNA in blood: Future genomic biomarkers for cancer detection. Experimental Hematology, 65, 17-28. doi: 10.1016/j.exphem.2018.06.003

6. Duffy, M. J. (2018). Circulating tumour DNA as a cancer biomarker. Annals of Clinical Biochemistry, 56(1), 42-48. Retrieved from https://doi.org/10.1177/000456321879840

7. Bolivar, A. M., Luthra, R., Mehrotra, M., Chen, W., Barkoh, B. A., Hu, P., Broaddus, R. R. (2019). Targeted next-generation sequencing of endometrial cancer and matched circulating tumor DNA: identification of plasma-based, tumor-associated mutations in early stage patients. Modern Pathologyvolume, 32, 405-414. doi: 10.1038/s41379-018-0158-8

8. Saba, R., Halytskyy, O., Saleem, N., \& Oliff, I. A. (2017). Buccal epithelium cigarette smoking, and lung cancer: review of the literature. Oncology, 93, 347-353. doi: $10.1159 / 000479796$

9. Bonassi, S., El-Zein, R., Bolognesi, C., \& Fenech M. (2011). Micronuclei frequency in peripheral blood lymphocytes and cancer risk: evidence from human studies. Mutagenesis, 26, 93-100. doi: 10.1093/mutage/geq075

10. Nersesyan, A., Fenech, M., Bolognesi, C., Mišík, M., Setayesh, T., Wultsch, G. ...Knasmüller, S. (2016). Use of the lymphocyte cytokinesis-block micronucleus as say in occupational biomonitoring of genome damage caused by in vivo exposure to chemical genotoxins: Past, present and future. Mutation Research, 770, 1-11. doi: 10.1016/j.mrrev.2016.05.003

11. Márcia Fernanda Correia Jardim Paz, Marcus Vinícius Oliveira Barros de Alencar, Antonio Luiz Gomes Junior, Keylla da Conceição Machado, Muhammad Torequl Islam, Eunus S. Ali,... Ana Amélia de Carvalho Melo-Cavalcante (2017). Correlations between Risk Factors for Breast Cancer and Genetic Instability in Cance Patients - A Clinical Perspective Study. Frontiers in Genetics, 8, 236. Retrieved from https://doi.org/10.3389/fgene.2017.00236

12. Kadhem, S. J., Ali, A. S. K., AL rikabi, \& Abdul-Ameer N. (2018). Compartment Between Breast Cancer Patient Undergoing Chemotherapy and Healthy Women by Cytokinesis - block Micronucleus testand Nuclear Division Index. Research Journal of Pharmacy and Technology, 11(2), 705. doi: 10.5958/0974-360X.2018.00133.6

13. Salimi, M. (2018). Comparative study of chemo-sensitivity expressed as micronuclei in lymphocytes of breast cancer patients, their unaffected first degree relatives and normal controls as a possible prognostic marker. International Journal of Radiation Research, 16(1), 85-93. Retrieved from http://ijrr.com/article-1-2145-en.html

14. Zölzer, F., Křižová, M., Freitinger Skalická Z., \& Rössnerová, A. (2017). Micronucleus frequency and content in healthy relatives of cancer patients. Biomarkers, 22 667-673. doi: 10.1080/1354750X.2016.1276627

15. Paltsev, M. A., Kvetnoy, I. M., Polyakova, V. O., Konovalov, S. S., Litvyakova, O. M., Linkova, N. S., ...Tolibova, G. K. (2012). Signaling molecules in buccal epithelium: optimization of diagnosis of socially significant diseases. Molecular Medicine 4,18-23. Retrieved from https://elibrary.ru/item.asp?id=18269445

16. Konovalov, S. S., Litviakova, O. M., Linkova, N. S., Sedov, E. V., Kvetnaya, T. V., Mursalov,S. U., Durnova, A. O., Tolibova, G. Kh., \& Kostylev, A. V. (2012). Immunocytochemistry of the buccal epithelium: optimisation of diagnostics of brest cancer. Molecula Medicine, 6, 57-59. Retrieved from https://elibrary.ru/item.asp?id=18736124

17. Rawat, A. \& Ganesh, N. (2018). Cytogenetic analysis of breast cancer females along with females with diabetes and thyroid issues to find a possible link Indian Journal of Scientific Research, 20(2), 329-334. Retrieved from https://www.researchgate.net/publication/329191104

18. Куртасова, Л. М., Савченко, А. А., Зуков, Р. А., \& Толмачева, Т. В. (2018) Ферментный спектр лимфоцитов периферической крови у больных почечно-клеточным раком до и после хирургического лечения. Меди и

19. Пелевина, И. И., Алещенко, А. В., Антощина, М. М., Бирюков, В. А. Карякин, О. Б., Кудряшова, О. В., ...Ярилин, А. А. (2014). Связаны ли свойства лимфоцитов периферической крови у больных раком предстательной железы с эффективностью лучевой терапии? Радиационная биология. Радиоэкология 54(30), 273-282. doi: 10.7868/S0869803114030126

20. Byakhova, M. M., Sycheva, L. P., Zhurkov, V. S., Selezneva, I. I., Kosmynin, A. A., Gabuniya, Z. R., ...Astrakhantsev, A. F. (2013). Cytogenetic status in patients with gastric cancer. Malignant Tumours, 3, 10-15. Retrieved from https://doi.org/10.18027/2224-5057-2013-3-10-15

21. Коленко, Ю. Г. (2015). Оценка эффективности микроядерного теста в клетках буккального эпителия для ранней диагностики лейкоплакии слизистой оболочки полости рта. Вісник проблем біології і медицини, 2(125), 353-358. Востановлена из https://cyberleninka.ru /article/n/otsenka-effektivnosti-mikroyadernogotesta-v-kletkah-bukkalnogo-epiteliya-dlya-ranney-diagnostiki-leykoplakii-slizistoyobolochki/viewer

22. Bolognesi, C., Bonassi, S., Knasmueller, S., Fenech, M., Bruzzone, M. Lando, C., ... Ceppi, M. (2015). Clinical application of micronucleus test in exfoliated buccal cells: A systematic review and metanalysis. Mutation Research, 766, 20-31. doi: 10.1016/j.mrrev.2015.07.002

23. Allione, A., Pardini, B., Viberti, C., Oderda, M., Allasia, M., Gontero, P., ...Matullo, G. (2018). The prognostic value of basal DNA damage level in peripheral blood lymphocytes of patients affected by bladder cancer. Urologic Oncology: Seminars and Original Investigations, 36(5), e15-e23. Retrieved from https://doi.org/10.1016/j.urolonc.2018.01.006

24. Buchynska, L. G., Brieieva, O. V., lurchenko, N. P., Protsenko, V. V., \& Nespryadko, S. V. (2017). DNA damage in tumor cells and peripheral blood lymphocytes of endometrial cancer patients assessed by the comet assay. Experimental Oncology, 39(4), 299-303. Retrieved from http://exp-oncology.com.ua/article /10578/dna-damagein-tumor-cells-and-peripheral-blood-lymphocytes-of-endometrial-cancer-patientsassessed-by-the-comet-assay
25. Amrin Shaikh, Darshana Barot, \& Divya Chandel. (2018). Genotoxic Effects of Exposure to Gasoline Fumes on Petrol Pump Workers. International Journal of Occupational and Environmental Medicine, 9(2), 79-87. doi: 10.15171/ijoem.2018.1159.

26. Pereira da Silva, V. H., de Luna Antonio, R., Pompeia, S., \& Ribeiro, D. A. (2015). Cytogenetic Biomonitoring in Buccal Mucosa Cells from Young Smokers. Acta Cytologica, 59, 474-478. doi: 10.1159/000443761

27. Ramesh, G., Chaubey, S., Raj, A., Seth, R. K., Katiyar, A., \& Kumar, A. (2017). Micronuclei assay in exfoliated buccal cells of radiation treated oral cancer patients. Journal of Experimental Therapeutics \& Oncology, 12(2), 121-128. Retrieved from https://www.ncbi.nlm.nih.gov/pubmed/29161779

28. Kesidi, S., Maloth, K. N., Kundoor Vinay Kumar Reddy, \& Geetha, P. (2017). Genotoxic and cytotoxic biomonitoring in patients exposed to full mouth radiographs A radiological and cytological study. Journal of Oral and Maxillofacial Radiology, 5, 1-6. doi: $10.4103 /$ jomr.jomr_47_16

29. Samuel Rangel Claudio, Jose Martim Marques Simas, Ana Carolina Flygare Souza, \& Maria do Carmo Baracho de Alencar. (2019). Genomic Instability and Cytotoxicity in Buccal Mucosal Cells of Workers in Banana Farming Evaluated by Micronucleus Test. Anticancer Research, 39(3), 1283-1286. doi: 10.21873/anticanres.13239

30. Sinitsky, M. Yu., Minina, V. I., Gafarov, N. I., Asanov, M. A., Larionov, A. V., Ponasenko, A. V., ...Druzhinin, V. G. (2016). Assessment of DNA damage in underground coal miners using the cytokinesis-block micronucleus assay in peripheral blood lymphocytes. Mutagenesis, 31(6), 669-675. Retrieved from https://doi.org/10.1093/mutage/gew038

31. Navas, A., Giraldo-Parra, L., Prieto M. D., Cabrera, J., \& Gómez, M. A. (2019). Phenotypic and functional stability of leukocytes from human peripheral blood samples: considerations for the design of immunological studies. BMC Immunology, 20(1), 5. doi: $10.1186 / \mathrm{s} 12865-019-0286-\mathrm{z}$

32. Gajski, G., Gerić, M., Oreščanin, V., \& Garaj-Vrhovac, V. (2018). Cytokinesisblock micronucleus cytome assay parameters in peripheral blood lymph ocytes of the general population: Contribution of age, sex, seasonal variations and lifestyle factors. ECotoxicologyand Environmental Safety, 148, 561-570. doi: 10.1016/j.ecoenv.2017.11.003

33. Guthrie, G. J., Charles, K. A. Roxburgh, C. S., Horgan, P. G. McMillan, D. C. \& Clarke, S. J. (2013). The systemic inflammation-based neutrophil-lymphocyte ratio: Experience in patients with cancer. Critical Reviews in Oncology/Hematology, 88(1), 218-230. doi: 10.1016/j.critrevonc.2013.03.010

34. Szkandera, J., Stotz, M., Eisner, F., Absenger, G., Stojakovic, T., Samonigg, H., ...Pichler, M. (2013). External Validation of the Derived Neutrophil to Lymphocyte Ratio as a Prognostic Marker on a Large Cohort of Pancreatic Cancer Patients. PLOS ONE, 8(11), e78225. doi: 10.1371/journal.pone.0078225

35. Sylman, J. L., Mitrugno, A., Atallah, M., Tormoen, G. W., Shatzel, J. J., Yunga, S. T., ...McCarty, O. J. T. (2018). The Predictive Value of Inflammation-Related Peripheral Blood Measurements in Cancer Staging and Prognosis. Frontiers in Oncology, 8, 78. doi: $10.3389 /$ fonc. 2018.00078

36. Fest, J., Ruiter, R., Mulder, M., Koerkamp, B. G., Ikram, M. A., Stricker, B. H., van Eijck, C. H. J. (2019). The systemic immune-inflammation index is associated with an increased risk of incident cancer - A population-based cohort study. International Journal of Cancer, 1-7. Retrieved from https://doi.org/10.1002/ijc.32303

37. Melahat Yildirim, Busra Demir Cendek, \& Ayse Filiz Avsar. (2015). Differentiation between benign and malignant ovarian masses in the preoperative period using neutrophil-to-lymphocyte and platelet-to-lymphocyte ratios. Molecular and Clinical Oncology, 3(2), 317-321. doi: 10.3892/mco.2014.481

38. Zhang, W. W., Liu, K. J., Hu, G. L., \& Liang, W. J. (2015). Preoperative platelet/lymphocyte ratio is a superior prognostic factor compared to other systemic inflammatory response markers in ovarian cancer patients. Tumour Biology, 36(11), 8831-8837. doi: 10.1007/s13277-015-3533-9

39. Çalışkan, S., Sungur, M., Kaba, S., Özsoy, E., Koca, O., \& Öztürk, M. İ. (2018). Neutrophil-to-Lymphocyte Ratio in Renal Cell Carcinoma Patients. Folia Medica (Plovdiv), 60(4), 553-557. doi: 10.2478/folmed-2018-0037

40. Stotz, M., Szkandera, J., Stojakovic, T., Seidel, J., Samonigg, H., Kornprat, P., Pichleret, M. (2015). The lymphocyte to monocyte ratio in peripheral blood represents a novel prognostic marker in patients with pancreatic cancer. Clinical Chemistry and Laboratory Medicine, 53(3), 499-506. doi:: 10.1515/cclm-2014-0447

41. Sun, W., Zhang, L., Luo, M., Hu, G., Mei, Q., Liu, D., ...Hu, G. (2016). Pretreatment hematologic markers as prognostic factors in patients with nasopharyngeal carcinoma: Neutrophil-lymphocyte ratio and platelet-lymphocyte ratio. Head \& Neck, 38, E1332-E1340. doi: 10.1002/hed.24224

42. Chan, J. C. Y., Chan, D. L., Diakos, C. I., Engel, A., Pavlakis, N., Gill, A Clarke, S. J. (2017). The Lymphocyte-to-Monocyte Ratio is a Superior Predictor of Overal I Survival in Comparison to Established Biomarkers of Resectable Colorectal Cancer. Annals of Surgery, 265(3), 539-546. doi: 10.1097/SLA.0000000000001743

43. Minardi, D., Scartozzi, M., Montesi, L., Santoni, M., Burattini, L., Bianconi, M., ...Muzzonigro, G. (2015). Neutrophil-to-lymphocyte ratio may be associated with the outcome in patients with prostate cancer. SpringerPlus, 4, 255 doi: 10.1186/s40064-015-1036-1

44. Cao, Z., Ji, J., Zhang, C., Wang, F., Xu, H., Yu, Y., \& Sun, Y. (2019). The preoperative neutrophil-to-lymphocyte ratio is not a marker of prostate can cer characteristics but is an independent predictor of biochemical recurrence in patients receiving radical prostatectomy. Cancer Medicine, 8(3), 1004-1012. doi: 10.1002/cam4.1984

45. Feng, Li, Haibo, Hu, Shuo Gu, Xin Chen, \& Qing Sun. (2015). Platelet to lymphocyte ratio plays an important role in prostate cancer's diagnosis and prognosis. International Journal of Clinical and Experimental Medicine, 8(7), 11746-11751. Retrieved from https://www.ncbi.nlm.nih.gov/pmc/articles/PMC4565397

46. Marín Hernández, C., Piñero Madrona, A., \& Gil Vázquez, P. J. (2018). Usefulness of lymphocyte-to-monocyte, neutrophil-to-monocyte and neutrophilto-lymphocyte ratios as prognostic markers in breast cancer patients treated with neoadjuvant chemotherapy. Clinical and Translational Oncology, 20(4), 476-483. doi: $10.1007 / \mathrm{s} 12094-017-1732-0$

47. Graziano, V., Grassadonia, A., lezzi, L., Vici, P., Pizzuti, L., Barba, M. Tinari, N. (2019). Combination of peripheral neutrophil-to-lymphocyte ratio and platelet-to-lymphocyte ratio is predictive of pathological complete response after neoadjuvant chemotherapy in breast cancer patients. The Breast, 44, 33-38. doi: 10.1016/j.breast.2018.12.014

48. Chang Ik Yoon, So Eun Park, Yoon Jin Cha, Soong June Bae, Chi Hwan Cha, DaYoung Lee, ...Joon Jeonget (2019). Associations between absolute neutrophil count and lymphocyte-predominant breast cancer. The Breast, 10. doi: 10.1016/j.breast.2019.09.013

49. Goel, A., \& Boland, C. R. (2012). Reviews in Basic and Clinical Gastroenterology and Hepatology Epigenetics of Colorectal Cancer. Gastroenterology, 143(6), 1442-1460. doi: 10.1053/j.gastro.2012.09.032 
50. Lu, Z., He, Q., Liang, J., Li, W., Su, Q., Chen, Z., ...Wang, A. (2019). miR-31-5p Is a Potential Circulating Biomarker and Therapeutic Target for Oral Cancer. Molecula Therapy - Nucleic Acids, 16, 471-480. doi: 10.1016/j.omtn.2019.03.012

51. Widschwendter, M., Jones, A., Evans, I., Reisel, D., Dillner, J., Sundström, K., ...Pashayan, N. (2018). Epigenome-based cancer risk prediction: rationale opportunities and challenges. Nature Reviews Clinical Oncology, 15, 292-309. doi: $10.1038 /$ nrclinonc. 2018.30

52. Koestler, D. C., Usset, J., Christensen, B. C., Marsit, C. J., Karagas, M. R., Kelsey, K. T., \&Wiencke, J. K. (2017). DNA Methylation-Derived Neutrophil-to-Lymphocyte Ratio: An Epigenetic Tool to Explore Cancer Inflammation and Outcomes. Cancer Epidemiology, Biomarkers \& Prevention, 26(3), 328-338. doi: 10.1158/1055-9965.EPI-16-0461

53. Wiencke, J. K., Koestler, D. C., Salas, L. A., Wiemels, J. L., Roy, R. P., Hansen, H. M., ...Christensen, B. C. (2017). Immunomethylomic approach to explore the blood neutrophil lymphocyte ratio (NLR) in glioma survival. Clinical Epigenetics, 9(10) 1-11. doi: 10.1186/s 13148-017-0316-8

54. Eljabo, N., Nikolic, N., Carkic, J., Jelovac, D., Lazarevic, M., Tanic, N., \& Milasin, J. (2018). Genetic and epigenetic alterations in the tumour, tumour margins, and normal buccal mucosa of patients with oral cancer. International Journal of Oral and Maxillofacial Surgery, 47(8), 976-982. doi: 10.1016/j.ijom.2018.01.020

55. Teschendorff, A. E., Yang, Z., Wong, A., Pipinikas, C. P., Jiao, Y., Jones, A., Widschwendter, M. (2015). Correlation of Smoking-Associated DNA Methylation Changes in Buccal Cells With DNA Methylation Changes in Epithelial Cancer. JAMA Oncology 1(4), 476-485. doi: 10.1001/jamaoncol.2015.1053

56. Bartel, D. P. (2009). MicroRNAs: target recognition and regulatory functions Cell, 136(2), 215-233. doi: 10.1016/j.cell.2009.01.002

57. Palkina, N., Komina, A., Aksenenko, M., Moshev, A., Savchenko, A., \& Ruksha, T. (2018). miR-204-5p and miR-3065-5p exert antitumor effects on melanoma cells. Oncology Letters, 15(6), 8269-8280. doi: 10.3892/ol.2018.8443

58. Farazi, T. A., Hoell, J. I., Morozov, P., \& Tuschl, T. (2013). MicroRNAs in human cancer. Advances in Experimental Medicine and Biology, 774, 1-20. doi: 10.1007/978-94-007-5590-1_1

59. Pedersen, N. J., Jensen, D. H., Lelkaitis, G., Kiss, K., Charabi, B. W., UIlum, H., ...von Buchwald, C. (2018). MicroRNA-based classifiers for diagnosis of ora cavity squamous cell carcinoma in tissue and plasma. Oral Oncology, 83, 46-52. doi: 10.1016/j.oraloncology.2018.05.020

60. Rapado-González, Ó., Majem, B., Muinelo-Romay, L., Álvarez-Castro, A., Santamaría, A., Gil-Moreno, A., ... \& Suárez-Cunqueiro M.M. (2018). Human salivary microRNAs in Cancer. Journal of Cancer, 9(4), 638-649. doi: 10.7150/jca.21180

61. Dou, Y-D., Huang, T., Wang, Q., Shu, X., Zhao, S.G., Li, L., ... Liu, H.B. (2018). Integrated microRNA and mRNA signatures in peripheral blood lymphocytes of familial epithelial ovarian cancer. Biochemical and Biophysical Research Communications, 496(1), 191-198. doi: 10.1016/j.bbrc.2018.01.023

62. Shu, L., Wang, Z., Wang, Q., Wang, Y., \& Zhang, X. (2018). Signature miRNAs in peripheral blood monocytes of patients with gastric or breast cancers. Open Biology, 8(10), 180051. doi: 10.1098/rsob. 180051

63. Chen, Z., Yu, T., Cabay, R. J., Jin, Y., Mahjabeen, I., Luan, X., \& Zhou, X. (2017). miR-486-3p, miR-139-5p, and miR-21 as Biomarkers for the Detection of Oral Tongue Squamous Cell Carcinoma. Biomarkers in Cancer, 9, 1-8. doi: 10.4137/BIC.S40981

64. Chang Chin-Wen, Wu Hui-Chen, Terry Mary Beth, \& Santella Regina M. (2015) microRNA Expression in Prospectively Collected Blood as a Potential Biomarker of Breast Cancer Risk in the BCFR. Anticancer research, 35(7), 3969-3977. Retrieved from https://www.ncbi.nlm.nih.gov/pmc/articles/PMC4776637

\section{Сучасний розвиток напрямку - цитологічна реактивність хворого онкологічного профілю}

Л.А. Налєскіна

Інститут експериментальної патології, онкології і радіобіологі ім. Р.Є. Кавецького НАН України, Київ

Резюме. В огляді проаналізовані дані літератури щодо підходів до оцінки реакції організму на пухлинний процес на рівні соматичних немалігнізованих клітин, які існують на сьогодні. Незважаючи на різний дизайн, метою всіх досліджень є пошук простих, неінвазивних і водночас інформативних маркерів, які можна було б використовувати в якості предикторів раку, для диференційної діагностики новоутворень, прогнозування пухлинного процесу, розробки заходів профілактики, оцінки ефективності лікування, а також визначення чутливості пухлини до хіміо- і променевої терапіі. Чітко простежуються три основні напрямки досліджень цитологічної реактивності хворого онкологічного профілю. Золотим стандартом залишається класичний підхід, що дозволяє в букальному епітелії або лімфоцитах периферичної крові оцінити цитогенетичні, цитотоксичні ефекти на підставі даних, отриманих під час дослідження аберацій хромосом, мікроядер, віднедавна ДНК-комет. У зв'язку з наявними доказами того, що ключовим фактором, який визначає результат злоякісного процесу, є запалення, широко розвивається другий напрямок цитологічної реактивності хворого онкологічного профілю - пошуки маркерів системної запальної відповіді на підставі індексів, розрахованих за співвідношенням формених елементів крові.
Віднедавна набув розвитку третій напрямок цієї проблеми епігенетичний, який полягає у вивченні маркерів метилування ДНК соматичних немалігнізованих клітин хворих зі злоякісними новоутвореннями різного генезу, зокрема лімфоцитів периферичної крові, а також мікроРНК, асоційованих з пухлинним ростом, для подальшого впровадження в клінічну практику. Наявні результати за всіма трьома напрямками досліджень дають надію на успішне вирішення в найближчій перспективі актуальних проблем онкології.

Ключові слова: цитологічна реактивність хворого онкологічного профілю; цитогенетичні, цитотоксичні ефекти; букальний епітелій, лімфоцити периферичної крові; мікроядра, системна запальна відповідь; метилування ДНК; мікроРНК.

\section{Analysis of cytologic reactivity of oncologic patient - modern trend in oncology \\ L.A. Naleskina}

R.E. Kavetsky Institute of Experimental Pathology, Oncology and Radiobiology, National Academy of Sciences of Ukraine, Kyiv

Summary. The review summarizes the modern literature data on the current approaches for the assessment of the host reaction to tumor at the level of somatic non-malignized cells. Notwithstanding the various designs of such studies, the general objective consists in the search for the simple non-invasive markers that may be used as the informative predictors of cancer for differential diagnosis and prognosis as well as the development of preventive measures, the assessment of treatment efficacy and the sensitivity of cancer cells to chemo- and radiotherapy. Three major directions in such studies may be delineated. The classical approach allowing for the analysis of cytotoxic effects based on the study of chromosomal aberrations, micronuclei and DNA comets in the samples of buccal epithelium and peripheral blood lymphocytes is still remaining as the golden standard. Since the inflammation is proved to be a key factor determining the outcome of the malignant process, the second direction in studying the cytological reactivity of cancer patients comprises the search for the markers of the systemic inflammatory response based on the indices calculated by the ratios of the peripheral blood cells. Recently, the epigenetic approach as the third research direction comprising this problem has been developed. Such an approach consists in the detection of the methylation markers in DNA of the somatic non-malignant cells, in particular in peripheral blood lymphocytes, as well as the analysis of tumor-associared microRNAs in the patients with malignant tumors of various genesis for their further clinical implementation. The results of the studies alongside all three directions delineated above are promising and offer the hopes for the success in the solution of the topical problems of cancer research in the near future.

Key words: cytological reactivity of cancer patient; cytogenetic, cytotoxic effects; buccal epithelium; peripheral blood lymphocytes; micronucleus; systemic inflammatory response; DNA methylation; microRNA.

Адрес:
Налескина Леся Анатольевна
03022, Киев, ул. Васильковская, 45
Институт экспериментальной патологии, онкологии и радиобиологии
$\begin{aligned} & \text { им. Р.Е. Кавецкого Национальной академии наук Украины } \\ & \text { Tел.: (044) 257-96-44 } \\ & \begin{array}{l}\text { E-mail: naleskina@ukr.net } \\ \text { Correspondence: } \\ \text { Naleskina Lesya }\end{array} \\ & \text { R.E. Kavetsky Institute of Experimental Pathology, } \\ & \text { Oncology and Radiobiology, National Academy of Sciences of Ukraine } \\ & \text { Tel.: (044) 257-96-44 } \\ & \text { E-mail: naleskina@ukr.net }\end{aligned}$

